\title{
Audit of the accuracy and remunerative implications of clinical coding in Elective Plastic Surgery
}

\author{
Andreas Georgiou ${ }^{1}$, Julia Ruston ${ }^{1}$, Shameem Haque ${ }^{1}$, and Alex Woollard ${ }^{1}$ \\ ${ }^{1}$ Royal Free London NHS Foundation Trust
}

September 21, 2020

\begin{abstract}
Introduction Accurate coding is essential to ensure remuneration for clinical procedures. However, coders are non-clinical and use operative and discharge notes to infer what they think to be appropriate codes. As plastic surgery encompasses a huge variety of procedures the codes used may not reflect the procedures performed. Method Electronic records from July/August 2019 were consulted to identify commonly performed procedures on elective lists which were then reviewed retrospectively by a coding auditor and a clinician. Procedures chosen to reflect a range of plastic surgery: 1) hands, 2) breast, 3) general plastics. The changes to the clinical codes (Procedural and Diagnostic) and HRG tariff implications were analysed. The audit loop was completed in July 2020 after the introduction of clinical coding improvement strategies. Results 65 relevant cases were included. Changes were made in 31 cases (24 OPCS- 4 code changes and 7 ICD-10 code changes). Significant remunerative implications for the department were noted (average $£ 209$ per patient). Conclusion This study demonstrated a clear need for collaboration between clinicians and coders. Increased surgeon awareness of clinical coding and clear documentation is essential. We have presented strategies of improving coding that is part of as part of a continued quality improvement programme.
\end{abstract}

Key Words: Clinical Audit, Public Health, Health Economics, Medical Informatics

\section{Introduction}

Clinical coding systems are used internationally across healthcare systems and are designed to bridge the gap between clinical practice and administration systems. The clinical codes assigned to patient episodes form the basis of reimbursement to the hospital, for treatments provided. Coding systems influence hospital and departmental budgets, medico-legal documentation and strategic planning at local and national levels ${ }^{1}$.Although a number of coding systems have been developed, the National Health Service (NHS) in the United Kingdom (UK) records surgical procedural data using the OPCS-4 (Office of Population Censuses and Surveys - Classification of Intervention and Procedures Version 4). Diagnostic data is coded using the ICD-10 (International Classification of Diseases Version 10). Clinically similar treatments which are felt to use similar levels of resources, are then grouped together as Healthcare Resource Groups (HRGs), which are allocated a fixed tariff. Clinical codes are therefore used to assign HRGs to patient encounters, which ultimately determine how much the hospital is paid for the care provided. The concept of using codes to generate tariffs, hence remunerating hospitals according to their activity levels, forms the basis of the Payment by Results (PbR) system introduced in 2004. ${ }^{2,3}$

In the UK, clinical coding is performed by health informatics personnel who are tasked to translate the medical terminology in a patient's medical records into a clinical code - in accordance to OPCS-4 codes and ICD-10 for diagnostic data. The coders are non-clinical and within the NHS may find themselves stretched in terms of resources and time. Plastic surgery is a diverse specialty which encompasses a huge range of diagnoses and procedures (often complex and multi-component) across the whole body. Evolving techniques, 
such as widespread adoption of microsurgery, have meant the field is ever-changing and abbreviations are also commonplace in operation notes. That there is a possibility of human error in assigning codes for these (often esoteric) cases is hardly surprising. Given that Plastic Surgery Departments are found in only a few tertiary centres, but often have large numbers of staff and generate a high number of patient care episodes, it is vital that codes are assigned accurately to ensure adequate remuneration for the work done.

We aimed to establish whether plastic surgery operations were being assigned the correct codes, and to determine the financial implications of any discordance. By identifying common errors and enhancing clinician collaboration with the coders, we attempted to improve the accuracy for the future and suggest suitable strategies for the long-term.

\section{Methods}

We liaised with three consultants and identified commonly performed elective plastic surgery procedures on their operating lists, classified as (Table 1): Hand Surgery, Breast Surgery and General Plastics.

We retrospectively examined the Consultants' elective cases during July and August 2019 and identified the 'commonly performed' procedures. The operation notes for these cases were reviewed with an experienced clinical coder and procedural (OPCS-4) and diagnostic (ICD-10) codes assigned. These were compared with the codes that had previously been assigned and submitted by the coding team, and any discrepancies noted. Any change in tariff that resulted from generating the new codes was also calculated.

Having identified areas of weakness within the coding strategy, we arranged regular meetings between clinicians and coders, to educate both parties as to the information required for accurate coding. Coders were provided with summaries of common operations and explanations of abbreviations and terminology. Clinicians were fed back which operations that had been documented less consistently and which areas required clarification in order to allocate codes which might have an impact financially. A repeat of our initial study was completed in July 2020, to ascertain whether these meetings had been effective. The clinical coding strategies employed are summarised in table 2 .

\section{Results}

65 cases were included for review, based on the list of commonly performed elective procedures in Table 1. (Fig. 1). Of the 65 cases reviewed, changes were made in 24 instances (36.9\%).

Errors were sub classified into four groups (Table 3):

A - Correct Codes assigned

B1 - Procedure code error or incompletely coded procedure (OPCS-4) code errors with no change in HRG

B2 - Procedure (OPCS-4) code error with a change in HRG

C1 - Diagnostic (ICD-10) code errors without a change in the HRG

C2 - Diagnostic (ICD-10) code errors with change to the HRG

In 10 of the 24 error cases, OPCS code changes resulted in HRG code changes (Table 3). When the new tariffs were calculated, and compared with those previously allocated, there was an increase in reimbursement to a value of $£ 13,593$. This is on average, an increase of $£ 209$ per patient. A change to the primary diagnosis was made in 7 cases, and of these, none resulted in HRG changes. Procedural Accuracy was found to be $63 \%$ and the diagnostic accuracy was $89.2 \%$. HRG accuracy was $84.6 \%$. An example of how coding errors were presented is shown in Table 4. It exemplifies how a revision operation can receive a significant amount of extra funding than a primary operation - yet this may not always be obvious to the coder reading an operation note. 
In July 2020, following the implementation of strategies described above (Table 2), 15 elective cases were reviewed. The initial OPCS codes were incorrect in 1 case (Procedural accuracy of 93\%). This resulted in no HRG code changes (HRG accuracy of 100\%). Reduced case numbers were due to elective operating restrictions during COVID-19.

\section{Discussion}

Most coders and clinicians never have any direct contact, nor do they know who to ask if questions arise. By introducing collaboration between the teams, and identifying points of contact, coding errors can be substantially reduced. The corollaries of accurate coding include fair remuneration, as well as improved service planning and provision.

Clinician involvement in clinical coding has previously been suggested in the literature as a way to improve the accuracy of clinical coding ${ }^{3,4,5,6,7,8}$.Across surgical specialties, whilst different procedures have been profiled, nearly every study found that funding was being lost to inaccurate procedural coding. Reconstructive specialties such as plastic surgery and oral and maxillofacial surgery have particular challenges due to the fact that one patient may undergo a multitude of procedures within one operation. Resection of primary malignancy, lymph node sampling and clearance, reconstruction with flaps and grafts, and secondary reconstruction of the donor site may all be involved, and each may need more than one OPCS-4 code. Extensive use of abbreviations makes describing such operations easier for the surgical team; however, coders may be unfamiliar with such specialised terminology. Naran et al. attribute this as one reason for $77 \%$ of oral surgery patients needing at least one coding amendment ${ }^{6}$. In our case, liaison with coders and providing reference and explanation of the commonly used abbreviations, helped save them time (searching internet browsers to decipher the notes) and improved coding accuracy. Previously, in cases where the abbreviation was not understood, the default was to assign a baseline procedural code e.g. 'other specified' or 'unspecified'.

Furthering the complexity of assigning codes, multiple surgical specialties may be involved in oncological cases (resection team and reconstruction team) as well as trauma (where bony fixation, internal injury management and soft tissue reconstruction may be required). Cooper et al. highlighted that in such cases, the primary admitting team may receive payment for the entire payment episode, whilst collaborating specialties receive none. ${ }^{9}$

The use of automated coding programmes seems to be a long way from clinical application, particularly in specialties such as Plastic Surgery, where procedures may be complex, idiosyncratic and regularly evolving ${ }^{10}$. However, simpler measures to try and standardise the pathway include the introduction of templates. Clinician-led coding (using templates) at the time of operation was found by Kannan et al. to increase tariffs in $49 \%$ of their general plastic surgery cases ${ }^{11}$. We suggest that all operating clinicians are educated as to which aspects of the operation/notes will have impact upon coding, so that they may ensure these are accurately documented. Templates and posters on display in theatre can assist with retaining and complying with this information (at least for commonly performed procedures, i.e. the majority of payments to be received).

Diagnostic codes are also important for tariffs. Certain diagnoses and comorbidities will result in changes to the HRG and final remuneration and should be documented at least on the patient discharge summary. This is not always known by clinicians and is sometimes neglected in the notes ${ }^{12}$.

A target of $100 \%$ data accuracy is the gold standard. ${ }^{13}$ Achieving 87\% (Audit Commission Report 2015) procedural and diagnostic accuracy is considered reasonable. ${ }^{14}$ these targets were met after the introduction of quality improvement strategies.

Coders will still ultimately be responsible for interpreting the operation notes and assigning the codes, and even with extra clinician awareness and conscientiousness when documenting procedures, some queries may still arise. We recommend close collaboration between clinical and coding team. In practical terms, it would be sensible for one clinician in the department to be assigned a coding liaison role. In our department, the 
coders now set aside any cases which have ambiguities and have regular meetings with a senior clinician to discuss these and clarify.

\section{Conclusion}

The importance of accurate, consistent and up-to-date clinical coding is undeniable. Our study suggests methods to improve coding accuracy; with education and templates for both clinicians and coders, and assigning a clinician to a coding liaison role. This can improve coding accuracy and increase subsequent remuneration for procedures. Adequate and fair reimbursement is essential to maintain the provision of high quality healthcare, especially when resources are finite and often stretched.

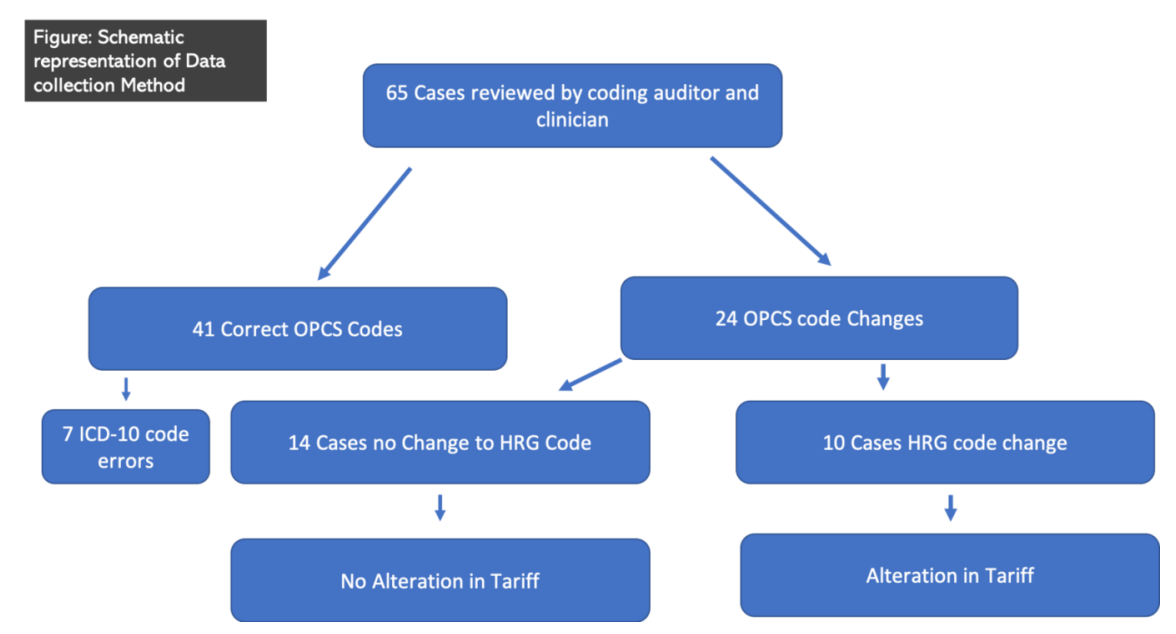

$\backslash$ protectFigure1

\section{References}

1.Burns EM, Rigby E, Mamidanna R, et al. Systematic review of discharge coding accuracy. J Public Health (Oxf) . 2012;34(1):138-148. doi:10.1093/pubmed/fdr054

2. Department of Health. A Simple Guide to Payment by Results. Leeds: DH; 2012

3. Wallis KL, Foxhall M, Warner RM, Jaffe W. Clinical coding inaccuracies in skin cancer surgery: the financial implications for a plastic surgery service. JPRAS2015 68(4) 582-3

4. Kannan RY, Neville C, Gwynn T, Venables V, Malhotra R, Nduka C. ' The effect of template-based sequential (TBS) coding on an NHS plastic Surgical practice' JPRAS 2018 Jul; 71(7):1058-1061

5. Heywood NA, Gill MD, Charlwood N, Brindle R, Kirwan CC 'Improving accuracy of clinical coding in surgery: collaboration is key'

J Surg Res 2016 Aug; 204(2):490-495

6. Naran S, Hudovsky A, Antscherl J, Howells S, Nouraei SAR ' Audit of accuracy of clinical coding in oral surgery' Br J Oral Maxilofac Surg 2014 Oct; 52(8):735-9 
7. Cheema ZA, Khwaja SA 'Implications of miscoding urological procedures in an era of financial austerity - 'every penny counts' JRSM Open 2015 Jul 7;6(6):2054270415593463

8. Britton E, Chambers C, Ashmore A ‘ orthopaedic procedure coding. Does accuracy matter?' Int J Health Care Qual Assur 209; 22(4):441-7

9. Cooper L, Stodell M, Payne C. Comment on 'Clinical coding inaccuracies in skin cancer surgery: the financial implications for a plastic surgery service.' JPRAS 2015 68(4) 584

10. Catling F, Spithourakis GP, Riedel S 'Towards automated clinical coding' International journal of medical informatics 2018 Dec; 120: 50-61

11. Kannan RY, Neville C, Gwynn T, Venables V, Malhotra R, Nduka C. ' The effect of template-based sequential (TBS) coding on an NHS plastic Surgical practice’ JPRAS 2018 Jul; 71(7):1058-1061

12.Mahbubani K, Georgiades F, Goh EL, et al. Clinician-directed improvement in the accuracy of hospital clinical coding. Future Healthc J . 2018;5(1):47-51. doi:10.7861/futurehosp.5-1-47

13. Colville RJ, Laing JH, Murison MS. Coding plastic surgery operations: an audit of performance using OPCS-4. Br J Plast Surg. 2000;53:420-2.

14.Audit Commission. Improving data quality in the NHS Annual report on the $\mathrm{PbR}$ assurance programme Health 2010 . http://www.auditcommission.gov.uk/SiteCollectionDocuments/Downloads/26082010pbrnhsdataqualityreport.pdf. $\quad$ May 2011, date last accessed.

\section{Hosted file}

Table 1.docx available at https://authorea.com/users/360024/articles/481763-audit-of-theaccuracy-and-remunerative-implications-of-clinical-coding-in-elective-plastic-surgery

\section{Hosted file}

Table 2.docx available at https://authorea.com/users/360024/articles/481763-audit-of-theaccuracy-and-remunerative-implications-of-clinical-coding-in-elective-plastic-surgery

\section{Hosted file}

Table 3.docx available at https://authorea.com/users/360024/articles/481763-audit-of-theaccuracy-and-remunerative-implications-of-clinical-coding-in-elective-plastic-surgery

\section{Hosted file}

Table 4.docx available at https://authorea.com/users/360024/articles/481763-audit-of-theaccuracy-and-remunerative-implications-of-clinical-coding-in-elective-plastic-surgery 


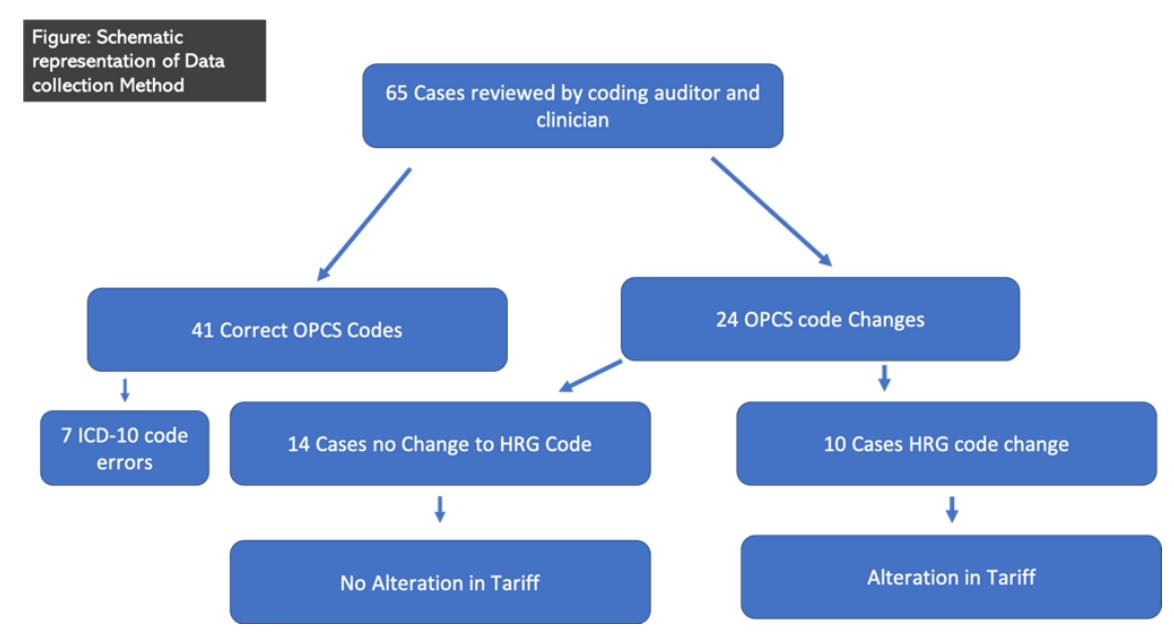

